The final journal version of this paper appears in Sendra J., Sendra J.R., (2014).

"Gröbner basis computation of Drazin inverses with multivariate

rational function entries". Applied Mathematics and Computation 259 (2015) 450-459.

and it is available at Elsevier via http://dx.doi.org/10.1016/j.amc.2015.02.070.

\title{
Gröbner Basis Computation of Drazin Inverses with Multivariate Rational Function Entries
}

\author{
Juana Sendra \\ Dpto. Matemática Aplicada a la I.T. de Telecomunicación. \\ Research Center on Software Technologies and Multimedia Systems \\ for Sustainability (CITSEM), UPM, Spain \\ jsendra@euitt.upm.es \\ J. Rafael Sendra \\ Dpto. de Fisica y Matemáticas, Universidad de Alcalá \\ E-28871 Madrid, Spain \\ rafael.sendra@uah.es
}

\begin{abstract}
In this paper we show how to apply Gröbner bases to compute the Drazin inverse of a matrix with multivariate rational functions as entries. When the coefficients of the rational functions depend on parameters, we give sufficient conditions for the Drazin inverse to specialize properly. In addition, we extend the method to weighted Drazin inverses. We present an empirical analysis that shows a good timing performance of the method.
\end{abstract}

keywords Drazin inverse, Gröbner bases, symbolic computation.

\section{Introduction}

Drazin inverses (introduced by M.P. Drazin in [6]) are used in many applications as singular differential equations and difference equations (see [4]), in finite finite Markov chains (see [16]), etc (see [1]). Many authors have addressed the problem of computing Drazin inverses where the matrix entries are indeed polynomials (see e.g. [2], [7], [10], [11]), for special type of matrices (see e.g. [15], [13]), and for weighted Drazin inverses (see [9], [12]). 
Some of the above quoted papers provide symbolic algorithms, in the sense that they use exact arithmetic and the output is exact, but techniques from computer algebra are not directly applied. In this paper, using that Drazin inverse computation can be translated into an elimination theory question, we show how to approach the problem by means of Gröbner bases (see e.g. [5] and [14]). Gröbner bases were introduced by B. Buchberger in [3] and, since then, they have been applied to multiple problems both in mathematics and in practical applications. Particularly important is the application of Gröbner bases to solve systems of algebraic equations. In this context, Gröbner bases can be seen as the generalization of gaussian elimination to the non-linear case of algebraic equations. The worst case complexity of Gröbner bases computation is double exponential in the number of variables, and simple exponential if the ideal is zero-dimensional (in the context of solving system of algebraic equations, it means that the number of solutions of the system is finite). Nevertheless, in practice, there are good implementations, and depending on the problem, the computation of a Gröbner basis is feasible.

In this paper, using Gröbner bases, we show how to compute the Drazin inverse of matrices which entries are rational functions in several variables. Moreover, we also deal with the problem when the input matrix has entries being rational functions in several variables with coefficients depending on parameters. In addition, we provide conditions to ensure when the Drazin inverse, of such parametric multivariate rational functions, specialized properly. We also present an empirical analysis that shows that the timings of the presented method has a good performance. We also show how these ideas apply to weighted Drazin inverses.

Throughout this paper we use the following notation. Let $\mathbb{F}$ be the rational function field $\mathbb{C}(\mathbf{z})$ (similarly, if we replace $\mathbb{C}$ by another field), where $\mathbf{z}=\left(z_{1}, \ldots, z_{s}\right)$, and let $\mathcal{M}_{n}(\mathbb{F})$ be the ring of $n \times n$ matrices over $\mathbb{F}$. For $A \in \mathcal{M}_{n}(\mathbb{F})$ we denote by $A^{D}$ the Drazin inverse of $A$ and by index(A) the Drazin index of $A$.

\section{The Multivariate Case}

Let $A \in \mathcal{M}_{n}(\mathbb{F})$ with Drazin index $k$. In order to approach the computation of the Drazin inverse $A^{D}$ of $A$, we consider a generic element $\hat{X}$ in $\mathcal{M}_{n}(\mathbb{F})$, that is $\hat{X}=$ $\left(x_{i j}\right)_{1 \leq i, j \leq n}$ where $x_{i j}$ are new variables. $A^{D}$ is the unique solution of the algebraic system of equations, in the variables $x_{i j}$,

$$
A^{k+1} \hat{X}-A^{k}=\mathbf{O}, \hat{X} A \hat{X}-\hat{X}=\mathbf{O}, A \hat{X}-\hat{X} A=\mathbf{O}
$$

where $\mathbf{O}$ is the null matrix. Therefore, if $\hat{\mathcal{F}}$ is set of polynomials of $\mathbb{F}[\mathbf{x}]$ (where $\mathbf{x}=$ $\left.\left(x_{11}, \ldots, x_{n n}\right)\right)$, provided by $(1)$, the ideal generated by $\hat{\mathcal{F}}$ over $\mathbb{F}[\mathbf{x}]$ is zero-dimensional, and since Gröbner bases algorithm does not extend the ground field, one concludes that $A^{D} \in \mathcal{M}_{n}(\mathbb{F})$. More generally, we have that following statement. 
Proposition 1 Let $\mathbb{L}$ be a field and $A \in \mathcal{M}_{n}(\mathbb{L})$, then $A^{D} \in \mathcal{M}_{n}(\mathbb{L})$.

We observe that $\mathcal{L}=\left\{A^{k+1} \hat{X}-A^{k}=\mathbf{O}, A \hat{X}-\hat{X} A=\mathbf{O}\right\}$ is a linear system of equations, while $\{\hat{X} A \hat{X}-\hat{X}=\mathbf{O}\}$ is an algebraic system of quadratic polynomials. Furthermore, $\mathcal{L}$ is compatible. Solving $\mathcal{L}$ and substituting the solution in the quadratic system we get a new quadratic (in general) system, equivalent to (1), and having less variables. Let

$$
X A X-X=\mathbf{O}
$$

be the resulting system. So let $\mathcal{F}$ be the set of polynomials defining (2); note that its ideal is zero-dimensional. The solution of a Gröbner basis of $\mathcal{F}$, w.r.t. a lexicographic order of its variables, jointly with the solution of $\mathcal{L}$, provides $A^{D}$. These ideas yield to the following algorithm.

Algorithm-1: Given $A \in \mathcal{M}_{n}(\mathbb{C}(\mathbf{z}))$ the algorithm computes its Drazin inverse $A^{D}$.

1. Compute $k:=\operatorname{index}(\mathrm{A})$.

2. Solve the linear system $\mathcal{L}=\left\{A^{k+1} \hat{X}-A^{k}=\mathbf{O}, A \hat{X}-\hat{X} A=\mathbf{O}\right\}$ and substitute its solution $\mathcal{S}$ in $\hat{X} A \hat{X}-\hat{X}=\mathbf{O}$. Let $X A X-X=\mathbf{O}$ be the resulting system and $V$ the set of variables.

3. Compute a Gröbner basis $\mathcal{G}$ of the polynomials defining $X A X-X=\mathrm{O}$ with respect to a lexicographic order of $V$.

4. Substitute the solution provided by $\mathcal{G}$ and $\mathcal{S}$ in $\hat{X}$ to get $A^{D}$.

Let us illustrate this by some examples.

Example 2 Let

$$
A=\left(\begin{array}{ccc}
\frac{1}{z_{2}} & z_{1} & 0 \\
0 & \frac{1}{z_{2}} & z_{1} \\
0 & 0 & 0
\end{array}\right) \in \mathcal{M}_{3}\left(\mathbb{C}\left(z_{1}, z_{2}\right)\right)
$$

$\operatorname{index}(\mathrm{A})=1$. The solution of $\mathcal{L}$ is

$$
\begin{gathered}
\left\{x_{1,1}=z_{2}, x_{1,2}=-z_{1} z_{2}{ }^{2}, x_{1,3}=x_{3,3} z_{1}{ }^{2} z_{2}{ }^{2}-2 z_{1}{ }^{2} z_{2}{ }^{3}, x_{2,1}=0, x_{2,2}=z_{2},\right. \\
\left.x_{2,3}=-z_{2} z_{1} x_{3,3}+z_{1} z_{2}{ }^{2}, x_{3,1}=0, x_{3,2}=0, x_{3,3}=x_{3,3}\right\}
\end{gathered}
$$

and the quadractic system (2) turns to be linear, indeed:

$$
\left\{x_{3,3}=0\right\}
$$

Therefore,

$$
A^{D}=\left(\begin{array}{ccc}
z_{2} & -z_{1} z_{2}{ }^{2} & -2 z_{1}{ }^{2} z_{2}{ }^{3} \\
0 & z_{2} & z_{1} z_{2}{ }^{2} \\
0 & 0 & 0
\end{array}\right) .
$$


Example 3 Let

$$
A=\left(\begin{array}{ccc}
z_{2} & 1 & 1 \\
z_{2} & z_{1}+1 & z_{1}+1 \\
-z_{2} & -z_{1}-1 & -z_{1}-1
\end{array}\right) \in \mathcal{M}_{2}\left(\mathbb{C}\left(z_{1}, z_{2}\right)\right)
$$

index $(\mathrm{A})=2$. The solution of $\mathcal{L}$ can be expressed as

$$
\begin{gathered}
\left\{x_{1,1}=z_{2}^{-1}, x_{1,2}=x_{1,2}, x_{1,3}=x_{1,2}, x_{2,1}=x_{1,2} z_{2}, x_{2,2}=-\frac{2 x_{1,2} z_{2}^{2}+x_{3,3} z_{2}-2}{z_{2}},\right. \\
\left.x_{2,3}=-\frac{x_{1,2} z_{2}^{2}+x_{3,3} z_{2}-1}{z_{2}}, x_{3,1}=-x_{1,2} z_{2}, x_{3,2}=\frac{x_{1,2} z_{2}^{2}+x_{3,3} z_{2}-1}{z_{2}}, x_{3,3}=x_{3,3}\right\}
\end{gathered}
$$

and the corresponding system (2) is

$$
\left\{\begin{array}{l}
-x_{1,2} z_{2}^{2}+1=0 \\
-x_{1,2} z_{1} z_{2}^{4}+2 x_{1,2} z_{1} z_{2}^{2}-x_{3,3} z_{2}^{2}-z_{1}-1=0 \\
-x_{1,2} z_{1} z_{2}^{4}+2 x_{1,2} z_{1} z_{2}^{2}-x_{1,2} z_{2}^{3}-x_{3,3} z_{2}^{2}-z_{1}+z_{2}-1=0 \\
x_{1,2}{ }^{2} z_{1} z_{2}^{4}-2 x_{1,2} z_{1} z_{2}^{2}+x_{1,2} z_{2}{ }^{3}+x_{3,3} z_{2}^{2}+z_{1}-z_{2}+1=0 \\
x_{1,2}{ }^{2} z_{1} z_{2}{ }^{4}-2 x_{1,2} z_{1} z_{2}{ }^{2}+2 x_{1,2} z_{2}^{3}+x_{3,3} z_{2}^{2}+z_{1}-2 z_{2}+1=0
\end{array}\right.
$$

that is quadratic in the variable $x_{1,2}, x_{3,3}$. A Gröbner basis of the above polynomials w.r.t. the lexicographic order, with $x_{1,2}<x_{3,3}$, is

$$
\left\{x_{1,2} z_{2}^{2}-1, x_{3,3} z_{2}^{2}+1\right\}
$$

that yields to

$$
A^{D}=\left(\begin{array}{ccc}
z_{2}^{-1} & z_{2}^{-2} & z_{2}^{-2} \\
z_{2}^{-1} & z_{2}^{-2} & z_{2}^{-2} \\
-z_{2}^{-1} & -z_{2}^{-2} & -z_{2}^{-2}
\end{array}\right)
$$

\section{The Parametric Multivariate Case}

We can use Gröbner bases for the computation of Drazin inverse of matrices with multivariate rational functions entries, which coefficients depend on parameters. More precisely, let $\Lambda$ be a finite set of parameters. We consider the matrix $\operatorname{ring} \mathcal{M}_{n}(\mathbb{C}(\Lambda)(\mathbf{z}))$. Let $A(\Lambda) \in \mathcal{M}_{n}(\mathbb{C}(\Lambda)(\mathbf{z}))$. We can deal with the Drazin inverse of $A(\Lambda)$; we denote it by $A(\Lambda)^{D}$. On the other hand, for every value $\Lambda_{0}$ of $\Lambda$ taken in $\mathbb{C}(\mathbf{z})$, we can deal with the Drazin inverse of $A\left(\Lambda_{0}\right)$; we denote it by $\left(A\left(\Lambda_{0}\right)\right)^{D}$. Algorithm-1 is valid for the field $\mathbb{C}(\Lambda)(\mathbf{z})$, and hence it computes $A(\Lambda)^{D}$. Also, Algorithm-1 is valid for the field 
$\mathbb{C}\left(\Lambda_{0}\right)(\mathbf{z})$, and so it also computes $\left(A\left(\Lambda_{0}\right)\right)^{D}$. The question is whether we can deduce $\left(A\left(\Lambda_{0}\right)\right)^{D}$ from $A(\Lambda)^{D}$; that is whether $A\left(\Lambda_{0}\right)^{D}=\left(A\left(\Lambda_{0}\right)\right)^{D}$. The next theorem states that for almost all $\Lambda_{0}$ the equality holds. We start with a lemma

Lemma 4 There exists a non-zero polynomial $H \in \mathbb{C}(\mathbf{z})[\Lambda]$ such that, if $\Lambda_{0} \in \mathbb{C}(\mathbf{z})$ satisfies that $H\left(\Lambda_{0}\right)$ is not identically zero, then $\operatorname{index}\left(\mathrm{A}\left(\Lambda_{0}\right)\right)=\operatorname{index}(\mathrm{A}(\Lambda))$.

Proof: Let $k=$ index $(\mathrm{A}(\Lambda))$ as matrix in $\mathcal{M}_{n}(\mathbb{C}(\Lambda)(\mathbf{z}))$. For $i=1, \ldots, k+1$, let $T_{i}$ denote the gaussian triangularization of $A^{i}$. Let us assume that we have expressed all non-zero entries of each $T_{i}$ with a common denominator. Then, $H$ can be taken as the product of the primitive part w.r.t. $\Lambda$ of the numerators and denominators of all diagonal elements of $T_{1}, \ldots, T_{k+1}$.

Theorem 5 There exists a non-zero polynomial $H \in \mathbb{C}(\mathbf{z})[\Lambda]$ such that, if $\Lambda_{0} \in \mathbb{C}(\mathbf{z})$ satisfies that $H\left(\Lambda_{0}\right)$ is not identically zero, then $A\left(\Lambda_{0}\right)^{D}=\left(A\left(\Lambda_{0}\right)\right)^{D}$.

Proof: For the theoretical reasoning, we consider that $A(\Lambda)^{D}$ is computed by taking a Gröbner basis of (1). Now, the proof follows from Lemma 4 and the behavior of Gröbner basis under specializations; see e.g. Exercise 7, p. 283 in [5].

The polynomial $H$ in Theorem 5 can be expressed as $H=H_{1} H_{2} H_{3}$ where:

- $H_{1}$ is the polynomial provided by the index computation (see Lemma 4),

- $\mathrm{H}_{2}$ is the primitive part, w.r.t. $\Lambda$, of the least common multiple of all denominators of the entries of $A(\Lambda)^{D}$, times the primitive part, w.r.t. $\Lambda$, of the least common multiple of all denominators of the entries of $A(\Lambda)$ (note that that the denominators in $A^{i}$ do not introduce different factors to those considered before), and

- $H_{3}$ is the polynomial provided by the Gröbner basis computation.

Although there exist algorithms to compute $H_{3}$, and hence to know in advance which evaluations are valid, these algorithm are time consuming. Nevertheless, in our case, since the solution is unique, we have a better result.

Theorem 6 Let $H_{1}, H_{2}$ as above. If $\Lambda_{0} \in \mathbb{C}(\mathbf{z})$ is such that $H_{1}\left(\Lambda_{0}\right) H_{2}\left(\Lambda_{0}\right) \neq 0$, then $A\left(\Lambda_{0}\right)^{D}=\left(A\left(\Lambda_{0}\right)\right)^{D}$.

Proof: Let $\Lambda_{0}$ be as in the statement. We consider the evaluation ring homomorphism $\varphi_{\Lambda_{0}}: \mathbb{C}[\Lambda, \mathbf{z}] \rightarrow \mathbb{C}[\mathbf{z}] ; \varphi(M(\Lambda, \mathbf{z}))=M\left(\Lambda_{0}, \mathbf{z}\right)$. We extend it to rational functions in $\mathbb{C}(\Lambda)(\mathbf{z})$ which denominators do not vanish at $\Lambda_{0}$, and similarly to $\mathcal{M}_{n}(\mathbb{C}(\Lambda)(\mathbf{z}))$. Because of $H_{1}$, index $\left(\varphi_{\Lambda_{0}}(\mathrm{~A}(\Lambda))\right)=\operatorname{index}\left(\mathrm{A}\left(\Lambda_{0}\right)\right)$ and $\varphi_{\Lambda_{0}}(A(\Lambda))=A\left(\Lambda_{0}\right), \varphi_{\Lambda_{0}}\left(A(\Lambda)^{k}\right)=$ 
$A\left(\Lambda_{0}\right)^{k}, \varphi_{\Lambda_{0}}\left(A(\Lambda)^{k+1}\right)=A\left(\Lambda_{0}\right)^{k+1}$, and because of $H_{2}, \varphi_{\Lambda_{0}}(A(\Lambda))^{D}=A\left(\Lambda_{0}\right)^{D}$. Therefore, $A\left(\Lambda_{0}\right)$ has index $k$ and $A\left(\Lambda_{0}\right)^{D}$ satisfies the equations

$$
A\left(\Lambda_{0}\right)^{k+1} X-A\left(\Lambda_{0}\right)^{k}=\mathbf{O}, X A\left(\Lambda_{0}\right) X-X=\mathbf{O}, A\left(\Lambda_{0}\right) X-X A\left(\Lambda_{0}\right)=\mathbf{O} .
$$

Now, the result follows from the uniqueness of the solutions of the system.

Remark 1 We observe that the method described in Theorem 6 is also applicable for matrices in $\mathcal{M}_{n}(\mathbb{C}(\mathbf{z}))$ considering $\mathbf{z}$ as parameters, and hence to determine, by specializations, the Drazin inverses of matrices in $\mathcal{M}_{n}(\mathbb{C})$.

Example 7 We consider Example 5.3 in [10]. There, the authors analyze the time for computing the Drazin inverse of the matrix

$$
B(q)=\left(\begin{array}{ccc}
1+z & z & 1+z \\
z^{q} & -1+z & z \\
1+z & 1 & 1+z
\end{array}\right) \in \mathcal{M}_{3}(\mathbb{C}(z))
$$

when $q \in \mathbb{N}$ takes natural values. We can consider the matrix

$$
A(\lambda)=\left(\begin{array}{ccc}
1+z & z & 1+z \\
\lambda & -1+z & z \\
1+z & 1 & 1+z
\end{array}\right) \in \mathcal{M}_{3}(\mathbb{C}(\lambda)(z))
$$

The matrix $(\lambda)$ is regular unless $\lambda=z$ (similarly, $B(q)$ is regular unless $q=1$ ), indeed,

$$
\operatorname{det}(A(\lambda))=-(1+z)(-1+z)(\lambda-z) .
$$

So, $H_{1}=\lambda-z$. The Drazin index is 0 and the Drazin inverse of $A(\lambda)$, as matrix in $\mathcal{M}_{3}(\mathbb{C}(\lambda, z))$, is

$$
A(\lambda)^{D}=\left(\begin{array}{ccc}
-\frac{z^{2}-z-1}{(1+z)(-1+z)(\lambda-z)} & (\lambda-z)^{-1} & -\frac{1}{(1+z)(-1+z)(\lambda-z)} \\
(-1+z)^{-1} & 0 & -(-1+z)^{-1} \\
-\frac{-z^{2}+\lambda+1}{(1+z)(-1+z)(\lambda-z)} & -(\lambda-z)^{-1} & \frac{z \lambda-z^{2}+1}{(1+z)(-1+z)(\lambda-z)}
\end{array}\right) .
$$

So, $H_{2}=(\lambda-z)$. Therefore, for $\lambda_{0} \in \mathbb{C}(z) \backslash\{z\}$ one has that $A\left(\Lambda_{0}\right)^{D}=\left(A\left(\Lambda_{0}\right)\right)^{D}$. For $\lambda_{0}=z$ the Drazin inverse is

$$
\left(\begin{array}{ccc}
-\frac{z^{3}-1}{\left(z^{2}-z-2\right)(1+z)(z-2)} & \frac{2 z^{2}-1}{(1+z)(z-2)^{2}} & -\frac{z^{3}-1}{z^{4}-2 z^{3}-3 z^{2}+4 z+4} \\
-\frac{z}{(1+z)(z-2)} & 2(z-2)^{-1} & -\frac{z}{(1+z)(z-2)} \\
\frac{2 z^{3}-2 z^{2}-z+1}{(z-2)\left(z^{3}-3 z-2\right)} & -\frac{3 z^{2}-z-3}{(1+z)(z-2)^{2}} & \frac{2 z^{3}-2 z^{2}-z+1}{\left(z^{2}-z-2\right)(1+z)(z-2)}
\end{array}\right)
$$

In particular, this implies that $A\left(z^{q}\right)^{D}=B(q)^{D}$ for any integer $q>1$. 
Example 8 Let

$$
A(\lambda)=\left(\begin{array}{cccc}
\lambda-z+1 & 1 & 0 & 0 \\
z-1-z^{-1} & -1+\lambda-z^{-1} & 0 & 0 \\
-\lambda+z^{-1}+z & -\lambda+z^{-1}+z & z & z \\
-z & -z & -z & -z
\end{array}\right) \in \mathcal{M}_{4}(\mathbb{C}(\lambda)(z))
$$

$\operatorname{index}(\mathrm{A}(\lambda))=2$ and

$$
A(\lambda)^{D}=\left(\begin{array}{cccc}
\frac{\lambda z-z-1}{(\lambda z-1)(\lambda-z)} & -\frac{z}{(\lambda z-1)(\lambda-z)} & 0 & 0 \\
-\frac{z^{2}-z-1}{(\lambda z-1)(\lambda-z)} & \frac{z(\lambda-z+1)}{(\lambda z-1)(\lambda-z)} & 0 & 0 \\
-\frac{z}{\lambda z-1} & -\frac{z}{\lambda z-1} & 0 & 0 \\
0 & 0 & 0 & 0
\end{array}\right)
$$

So $H_{2}=(\lambda z-1)(\lambda-z)$. The triangularization $T_{i}$ of $A^{i}, i=1,2,3$ are

$$
\begin{gathered}
T_{1}=\left(\begin{array}{cccc}
-z & -z & -z & -z \\
0 & \lambda-z & -\frac{z^{2}-z-1}{z} & -\frac{z^{2}-z-1}{z} \\
0 & 0 & \frac{\lambda z-1}{z} & \frac{\lambda z-1}{z} \\
0 & 0 & 0 & 0
\end{array}\right), T_{2}=\left(\begin{array}{cccc}
-\frac{(\lambda z-1)^{2}}{z^{2}} & -\frac{(\lambda z-1)^{2}}{z^{2}} & 0 & 0 \\
0 & (\lambda-z)^{2} & 0 & 0 \\
0 & 0 & 0 & 0 \\
0 & 0 & 0 & 0
\end{array}\right) \\
T_{3}=\left(\begin{array}{cccc}
-\frac{(\lambda z-1)^{3}}{z^{3}} & -\frac{(\lambda z-1)^{3}}{z^{3}} & 0 & 0 \\
0 & (\lambda-z)^{3} & 0 & 0 \\
0 & 0 & 0 & 0 \\
0 & 0 & 0 & 0
\end{array}\right) .
\end{gathered}
$$

Thus, $H_{1}=(\lambda-z)(\lambda z-1)$. Therefore, for $\lambda_{0} \in \mathbb{C}(z) \backslash\left\{z, z^{-1}\right\}$, one has that $A\left(\lambda_{0}\right)^{D}=$ $\left(A\left(\lambda_{0}\right)\right)^{D}$. For $\lambda_{0}=z$ or $\lambda_{0}=z^{-1}$ one can apply Algorithm-1.

\section{Weighted Drazin Inverses}

The ideas developed in Sections 2 and 3 can be extended to the case of weighted Drazin inverses (see [9]). More precisely, let $A \in \mathcal{M}_{n \times m}(\mathbb{F})$ and $W \in \mathcal{M}_{m \times n}(\mathbb{F})$ and let $k=\operatorname{index}(\mathrm{AW})$. In order to approach the computation of the $W$-Weighted Drazin inverse $A^{D, W}$ of $A$, we consider a generic element $\hat{X}$ in $\mathcal{M}_{n \times m}(\mathbb{F})$, that, is 
$\hat{X}=\left(x_{i j}\right)_{1 \leq i \leq n, j \leq m}$ where $x_{i j}$ are new variables. $A^{D, W} \in \mathcal{M}_{n \times m}(\mathbb{F})$ is the unique solution of the algebraic system of equations, in the variables $x_{i j}$,

$$
(A W)^{k+1} \hat{X} W-(A W)^{k}=\mathbf{O}, \hat{X} W A W \hat{X}-\hat{X}=\mathbf{O}, A W \hat{X}-\hat{X} W A=\mathbf{O}
$$

where $\mathbf{O}$ is the null matrix. Reasoning as in Proposition 1, one deduces that

Proposition 9 Let $\mathbb{L}$ be a field and $A \in \mathcal{M}_{n \times m}(\mathbb{L}), W \in \mathcal{M}_{m \times n}(\mathbb{L})$, then $A^{D, W} \in$ $\mathcal{M}_{n \times m}(\mathbb{L})$.

Reasoning as in Section 2, we get the following algorithm.

Algorithm-1W: $A \in \mathcal{M}_{n \times m}(\mathbb{C}(\mathbf{z}))$ and $W \in \mathcal{M}_{m \times n}(\mathbb{C}(\mathbf{z}))$ the algorithm computes its Drazin weighted inverse $A^{D, W}$.

1. Compute $k:=$ index $(\mathrm{AW})$.

2. Solve the linear system $\mathcal{L}=\left\{(A W)^{k+1} \hat{X} W-(A W)^{k}=\mathbf{O}, A W \hat{X}-\hat{X} A W=\mathbf{O}\right\}$ and substitute its solution $\mathcal{S}$ in $\hat{X} W A W \hat{X}-\hat{X}=\mathbf{O}$. Let $X W A W X-X=\mathbf{O}$ be the resulting system and $V$ the set of variables.

3. Compute a Gröbner basis $\mathcal{G}$ of the polynomials defining $X W A W X-X=\mathbf{O}$ with respect to a lexicographic order of $V$.

4. Substitute the solution provided by $\mathcal{G}$ and $\mathcal{S}$ in $\hat{X}$ to get $A^{D, W}$.

Example 10 We consider the matrices

$$
A=\left(\begin{array}{cccc}
z_{1} & z_{1} & z_{1} & z_{1} \\
z_{2} & z_{2} & z_{2} & z_{2} \\
1 & 1 & 1 & 1
\end{array}\right), W=\left(\begin{array}{ccc}
z_{2}^{-1} & z_{2} & z_{2} \\
0 & 0 & 0 \\
0 & 0 & 0 \\
0 & 0 & 0
\end{array}\right)
$$

The index of $A W$ is 1. Applying Algorithm1 $W$ we get

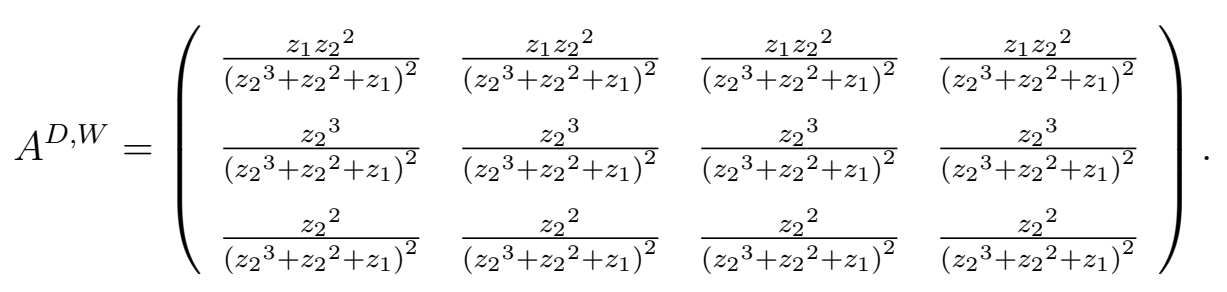




\section{$5 \quad$ Empirical Analysis}

The complexity for computing Gröbner bases is, in general, double exponential. In our case, since the ideal is zero-dimensional, the worst case complexity is $2^{\mathcal{O}\left(n^{2}-r\right)}$, where $r$ is the rank of the matrix associated with the linear system $\mathcal{L}$ (see Theorem 3 in [8]). Nevertheless, in this section we show by means of some empirical experiments that the actual computing time of the algorithm has a good time performance. We consider three experiments. In the first one, the number of variable $z_{i}$ is fixed, in fact 2 , however, the order of the matrix, as well as the degree of its rational function entries, increase. In the second experiment, the degree of the rational function entries is fixed, in fact 2 , however, the order of the matrix, as well as the number of variables, increase. The third experiment is similar to the second by neither the number of variables nor the degree is fixed. The computations has been done on Intel(R) Core(TM) i7-2630QM at $2.00 \mathrm{GHz} 16 \mathrm{~GB}$. Time is measured in second and the computations have been executed using the mathematical software Maple 17.

\section{First experiment}

We consider matrices constructed as follows. Let $J_{\rho, \ell}$ be the $\ell \times \ell$ Jordan block of $\rho \in$ $\mathbb{C}(\mathbf{z})$; with 1 over the diagonal. Then, we take $A\left(n_{1}, n_{2}\right)=Q \cdot P \in \mathcal{M}_{n_{1}+n_{2}}\left(\mathbb{C}\left(z_{1}, z_{2}\right)\right)$ where

$$
P=\left(\begin{array}{cc}
J_{z_{2}, n_{1}} & M \\
\mathrm{O} & J_{0, n_{2}}
\end{array}\right),
$$

$M=\left(m_{i, j}\right)$ is such that $m_{i, j}=1, \forall i, j$, and $Q=\left(q_{i, j}\right) \in \mathcal{M}_{n_{1}+n_{2}}(\mathbb{C}(\mathbf{z}))$ is such that $q_{i, i}=0, q_{i, j}=i / z_{2}$ if $i<j$ and $q_{i, j}=j z_{1} z_{2}$ if $i>j$. For instance, for $n_{1}=n_{2}=2$ we have

$$
P=\left(\begin{array}{cccc}
z_{1} & 1 & 1 & 1 \\
0 & z_{1} & 1 & 1 \\
0 & 0 & 0 & 1 \\
0 & 0 & 0 & 0
\end{array}\right), Q=\left(\begin{array}{cccc}
0 & 2 z_{2} z_{1} & 3 z_{2} z_{1} & 4 z_{2} z_{1} \\
2 z_{2}^{-1} & 0 & 3 z_{2} z_{1} & 4 z_{2} z_{1} \\
3 z_{2}^{-1} & 3 z_{2}^{-1} & 0 & 4 z_{2} z_{1} \\
4 z_{2}^{-1} & 4 z_{2}^{-1} & 4 z_{2}^{-1} & 0
\end{array}\right)
$$

and

$$
A(2,2)=\left(\begin{array}{cccc}
0 & 2 z_{1}^{2} z_{2} & 2 z_{2} z_{1} & 5 z_{2} z_{1} \\
2 \frac{z_{1}}{z_{2}} & 2 z_{2}^{-1} & 2 z_{2}^{-1} & 2 z_{2}^{-1}+3 z_{2} z_{1} \\
3 \frac{z_{1}}{z_{2}} & 3 z_{2}^{-1}+3 \frac{z_{1}}{z_{2}} & 6 z_{2}^{-1} & 6 z_{2}^{-1} \\
4 \frac{z_{1}}{z_{2}} & 4 z_{2}^{-1}+4 \frac{z_{1}}{z_{2}} & 8 z_{2}^{-1} & 12 z_{2}^{-1}
\end{array}\right) .
$$

Table 5 shows the computing times for determining $A(i, j)^{D}$ when $i, j \in\{1,2,3,4\}$, as well as the degree and number of terms in $A(i, j)^{D}$. More precisely, if $A(i, j)^{D}=$ $\left(\delta_{k_{1}, k_{2}}\right)_{1 \leq k_{1}, k_{2} \leq i+j} \in \mathcal{M}_{i+j}(\mathbb{C}(\mathbf{z}))$ then we denote by $\operatorname{deg}\left(A_{i, j}^{D}\right)=\max \left\{\operatorname{deg}_{\mathbf{z}}\left(\delta_{k_{1}, k_{2}}\right) \mid 1 \leq\right.$ $\left.k_{1}, k_{2} \leq i+j\right\}$, where the degree of a rational function is the maximum of its numerator 
degree and its denominator degree. Also, we denote by $\operatorname{terms}\left(A(i, j)^{D}\right)$ the maximum number of non-zero terms in the rational functions entries of $A(i, j)^{D}$.

\begin{tabular}{|c||c|c|c|c|c||c|c|c|c|}
\hline & time & $N_{1}$ & $N_{2}$ & $N_{3}$ & & time & $N_{1}$ & $N_{2}$ & $N_{3}$ \\
\hline \hline$(1,1)$ & 0.078 & $2 \times 2$ & 2 & 3 \\
$(1,2)$ & 0.063 & $3 \times 3$ & 8 & 6 \\
$(1,3)$ & 0.140 & $4 \times 4$ & 12 & 10 \\
$(1,4)$ & 0.281 & $5 \times 5$ & 18 & 14 \\
$(2,1)$ & 0.078 & $3 \times 3$ & 18 & 14 \\
$(2,2)$ & 0.281 & $4 \times 4$ & 18 & 25 \\
$(2,3)$ & 0.951 & $5 \times 5$ & 20 & 33 \\
$(2,4)$ & 2.215 & $6 \times 6$ & 26 & 39 \\
\hline
\end{tabular}

Table 1: Results of Experiment 1: $N_{1}=\operatorname{order}(A(i, j)), N_{2}=\operatorname{deg}\left(A_{i, j}^{D}\right), N_{3}=$ $\operatorname{terms}\left(A(i, j)^{D}\right)$

\section{Second experiment}

Given natural numbers $n, m$, with $n>2$, we consider matrices constructed as follows. Let $\Delta_{n-2}=\left(\delta_{i, j}\right)$ be the $(n-2) \times(n-2)$ matrix where

$$
\delta_{i, j}=i \sum_{\ell=1}^{m} z_{\ell}+\frac{j}{z_{2}}
$$

and let $Q=\left(q_{i, j}\right)$ be the $n \times n$ matrix where

$$
q_{i, j}=-1 \text { if } i>j \text { and } q_{i, j}=1 \text { if } 1 \leq j .
$$

Then, we take

$$
A(m, n)=Q \cdot\left(\begin{array}{cc}
\Delta_{n-2} & \mathrm{O} \\
\mathbf{O} & J_{0,2}
\end{array}\right) \cdot Q^{-1} \in \mathcal{M}_{n}\left(\mathbb{C}\left(z_{1}, \ldots, z_{m}\right)\right)
$$

with $J_{0,2}$ the $2 \times 2$ Jordan block associated with 0 . Table 5 shows the computing times of determining $A(i, j)^{D}$ when $i \in\{1,2,3,4,5,6,7,8,9,10\}$ and $j \in\{3,4,5\}$, as well as the number of variables and terms $\left(A(i, j)^{D}\right)$; note that the degree here is always 2 .

\section{Third experiment}

We consider the same construction as in the previous experiment, but taking

$$
\delta_{i, j}=i \sum_{\ell=1}^{m} z_{\ell}^{i}+\frac{j}{z_{2}} .
$$

Table 5 shows the results of the experiment.

Acknowledgement: Authors supported by the Spanish Ministerio de Economía y Competitividad under the Project MTM2011-25816-C02-01. The authors are members of the Research Group ASYNACS (Ref. CCEE2011/R34) 


\begin{tabular}{|c||c|c|c|c|c||c|c|c|c|}
\hline & time & $N_{1}$ & $N_{2}$ & $N_{3}$ & & time & $N_{1}$ & $N_{2}$ & $N_{3}$ \\
\hline \hline$(1,3)$ & 0.078 & $3 \times 3$ & 2 & 4 & $(6,3)$ & 0.125 & $3 \times 3$ & 6 & 13 \\
$(1,4)$ & 0.156 & $4 \times 4$ & 2 & 4 & $(6,4)$ & 0.421 & $4 \times 4$ & 6 & 13 \\
$(1,5)$ & 0.422 & $5 \times 5$ & 2 & 4 & $(6,5)$ & 2.902 & $5 \times 5$ & 6 & 13 \\
$(2,3)$ & 0.063 & $3 \times 3$ & 2 & 5 & $(7,3)$ & 0.156 & $3 \times 3$ & 7 & 13 \\
$(2,4)$ & 0.172 & $4 \times 4$ & 2 & 5 & $(7,4)$ & 0.515 & $4 \times 4$ & 7 & 15 \\
$(2,5)$ & 0.702 & $5 \times 5$ & 2 & 5 & $(7,5)$ & 7.629 & $5 \times 5$ & 7 & 15 \\
$(3,3)$ & 0.094 & $3 \times 3$ & 3 & 6 & $(8,3)$ & 0.156 & $3 \times 3$ & 8 & 15 \\
$(3,4)$ & 0.281 & $4 \times 4$ & 3 & 7 & $(8,4)$ & 0.577 & $4 \times 4$ & 8 & 17 \\
$(3,5)$ & 1.076 & $5 \times 5$ & 3 & 7 & $(8,5)$ & 6.880 & $5 \times 5$ & 8 & 17 \\
$(4,3)$ & 0.094 & $3 \times 3$ & 4 & 7 & $(9,3)$ & 0.171 & $3 \times 3$ & 9 & 17 \\
$(4,4)$ & 0.281 & $4 \times 4$ & 4 & 7 & $(9,4)$ & 0.765 & $4 \times 4$ & 9 & 19 \\
$(4,5)$ & 5.039 & $5 \times 5$ & 4 & 9 & $(9,5)$ & 13.869 & $5 \times 5$ & 9 & 19 \\
$(5,3)$ & 0.125 & $3 \times 3$ & 5 & 11 & $(10,3)$ & 0.203 & $3 \times 3$ & 10 & 19 \\
$(5,4)$ & 0.343 & $4 \times 4$ & 5 & 11 \\
$(5,5)$ & 1.716 & $5 \times 5$ & 5 & 11 \\
\hline & $(10,4)$ & 0.858 & $4 \times 4$ & 10 & 21 \\
$(10,5)$ & 23.307 & $5 \times 5$ & 10 & 21 \\
\hline
\end{tabular}

Table 2: Results of Experiment 2: $N_{1}=\operatorname{order}(A(i, j)), N_{2}=$ Number of variables, $N_{3}=\operatorname{terms}\left(A(i, j)^{D}\right)$

\section{References}

[1] Ben-Israel A., Greville T.N.E., Generalized Inverses: Theory and Applications, second ed., Springer-Verlag, Berlin, 2003.

[2] Bu F., Wei Y. The algorithm for computing the Drazin inverses of two-variable polynomial matrices. Applied Mathematics and Computation 147 (2004) 805-836

[3] Buchberger B., (1970) Ein Algoritmus zum Baseselemente de Restklassenringen nach einen nulldimensionen Polynomideal, Ph. D. Thesis Math. Inst., Univ. of Innsbruk, Austria (1965) y Aequationes Math. n. 4, 3, pp 374-383 (1970)

[4] Campbell S.L., Meyer C.D., Rose N.J. Applications of the Drazin inverse to linear systems of differential equations with singular constant coefficients, SIAM J. Appl. Math. 31 (1976) 411-425.

[5] Cox D., Little J. and O'Shea D. (1997). Ideals, Varieties, and Algorithms. SpringerVerlag, New York.

[6] Drazin M.P. Pseudo inverse in associative rings and semigroups, Amer. Math. Monthly 65 (1958) 506-514.

[7] Ji J. A finite algorithm for the Drazin inverse of a polynomial matrix. Applied Mathematics and Computation 130 (2002) 243-251. 


\begin{tabular}{|c||c|c|c|c|c|c||c|c|c|c|c|}
\hline & time & $N_{1}$ & $N_{2}$ & $N_{3}$ & $N_{4}$ & & time & $N_{1}$ & $N_{2}$ & $N_{3}$ & $N_{4}$ \\
\hline \hline$(1,3)$ & 0.062 & $3 \times 3$ & 2 & 4 & 2 & $(3,3)$ & 0.094 & $3 \times 3$ & 3 & 34 & 7 \\
$(1,4)$ & 0.218 & $4 \times 4$ & 2 & 5 & 3 & $(3,4)$ & 0.328 & $4 \times 4$ & 3 & 34 & 7 \\
$(1,5)$ & 1.014 & $5 \times 5$ & 2 & 11 & 6 & $(3,5)$ & 62.603 & $5 \times 5$ & 3 & 71 & 7 \\
$(2,3)$ & 0.063 & $3 \times 3$ & 2 & 11 & 6 & $(4,3)$ & 0.093 & $3 \times 3$ & 4 & 71 & 7 \\
$(2,4)$ & 0.249 & $4 \times 4$ & 2 & 11 & 6 & $(4,4)$ & 0.437 & $4 \times 4$ & 4 & 71 & 7 \\
$(2,5)$ & 3.276 & $5 \times 5$ & 2 & 34 & 7 & $(4,5)$ & 185.751 & $5 \times 5$ & 4 & 121 & 7 \\
\hline
\end{tabular}

Table 3: Results of Experiment 3: $N_{1}=\operatorname{order}(A(i, j)), N_{2}=$ Number of variables, $N_{3}=\operatorname{terms}\left(A(i, j)^{D}\right), N_{4}=\operatorname{deg}\left(A(i, j)^{D}\right)$.

[8] Lakshman Y.N. 1990. A single exponential bound on the complexity of computing Gröbner bases of zero dimensional ideals, in Effective Methods in Algebraic Geometry (Proc. MEGA-90). T.Mora, C.Traverso (eds.), Progress in Mathematics 94, 227-234, Birkhäuser

[9] Stanimirović P.S, Cvetković-Ilić D.S. Successive matrix squaring algorithm for computing outer inverses. Applied Mathematics and Computation 203 (2008) 19-29.

[10] Stanimirović P.S, Milan B. Tasić M.B., Vu K.M. Extensions of Faddeevs algorithms to polynomial matrices Applied Mathematics and Computation 214 (2009) 246-258.

[11] Tasić M.B., Stanimirović P.S. Symbolic and recursive computation of different types of generalized inverses. Applied Mathematics and Computation 199 (2008) 349-367

[12] Tasić M.B., Stanimirović P.S., Petković M.D., Symbolic computation of weighted MoorePenrose inverse using partitioning method. Applied Mathematics and Computation 189 (2007) 615-640

[13] Wei Y., Diao H., Ng M. On Drazin inverse of singular Toeplitz matrix. Applied Mathematics and Computation 172 (2006) 809-817.

[14] Winkler F., (1996). Polynomial Algorithms in Computer Algebra. Springer-Verlag, Wien New York.

[15] Wülling W. The Drazin inverse of a singular, unreduced tridiagonal matrix. Linear Algebra and its Applications 439 (2013) 2736-2745.

[16] Zhang X., Chen G. The computation of Drazin inverse and its application in Markov chains. Applied Mathematics and Computation 183 (2006) 292-300 